\title{
Papers
}

\section{Marty Carroll}

is director of usability practice at The Usability Company. With many years of usability experience and a background in psychology, his role involves applying usability methods to deliver against business objectives for large blue-chip organisations.
Keywords: usability, ROI, Web analytics, Web metrics
Marty Carroll

The Usability Company

The Lightwell

12-16 Laystall Street

London EC1R 4PF, UK

Tel: +44 (0)20 78436707 Mobile: +44 (0)7970 744415

Fax: +44 (0)20 78436701

E-mail:

marty@theusabilitycompany.com

\section{Usability and Web analytics: ROI justification for an Internet strategy}

\author{
Marty Carroll \\ Received (in revised form): 7 October 2002
}

\begin{abstract}
Usability is becoming an increasingly critical issue for commercial websites. Even big brand names are losing substantial revenues because their sites are too complicated for customers. Traditionally, usability was simply about making life easier for customers rather than impacting on the bottom line. When combined with appropriate measuring tools and metrics, usability has a return on investment (ROI) demonstrably greater than any other initiative. Making a site easier to use means that it is easier for customers to do business with the company.

This paper proposes an approach whereby an Internet strategy based on performance measurement and a focus on usability offers an unequivocal ROI. The demonstrable ROI lends credibility to the business justification for an Internet strategy or augmentation of an existing strategy.

This approach involves determining key business metrics and developing a solution employing usability methods to benchmark, analyse, resolve and remeasure to position a site that delivers on commercial objectives. This strategy is not for the faint of heart, but it does help to increase revenues and reduce costs while offering a sustainable competitive advantage.
\end{abstract}

\section{Introduction}

The dot.com boom and subsequent implosion should have taught many a hard lesson about the flippancy with which cash was burned on e-business initiatives. However, even today many organisations are forging ahead with plans for new sites or site changes with ill-defined goals and very little consideration of the ROI. The relationship between budget spend (whether on a new site or amending an existing site) and commercial benefit is not at all clear. Decisions to allocate budgets are based more on intuition or vague market research than on a patent understanding of the ROI.

\section{The need for accountability}

While there is a tacit acknowledgment that the Internet is a core business platform for many organisations, the success of this channel is not subjected to the same stringent success criteria applied to other media. There is a shift occurring whereby a new level of accountability is being 


\section{No informed decision making}

demanded. In a climate where the focus is necessarily on the bottom line and with executives taking a tentative approach to the allocation of budgets, there is a requirement for a clear and demonstrable ROI.

Managers have been precluded from informed decision making in the past because of the lack of a programme geared to understanding how successful (if at all) the site is numerically. If an organisation is not gauging the success of its website(s) in terms of numbers it is doing itself a great disfavour. A framework for measuring success needs to be introduced. Adopting this kind of programme is a significant step in the vindication of budget spend and the realisation of true business benefit.

\section{ROI eluding organisations}

There are two primary reasons why businesses have not implemented such a strategy. The first is because managers simply could not or did not know how to avail themselves of the data providing performance indicators for the site. Secondly, having obtained an understanding of the performance of the site, the data intelligence to inform design decisions has been lacking. Below the reasons why performance indicators have been so elusive are outlined, and later the paper discusses how the intelligence can be gathered through a strategic focus on usability.

A recent Forrester report found that 90 per cent of site managers believe that measuring the business goals of their site(s) is 'extremely or very important'. 'With an increased importance attached to these measurements, why is it that very few organisations are actually measuring performance? Essentially there are seven principal reasons.

- The data reside on different systems or are generated by different tools, and this causes issues in the amalgamation of the data to obtain a complete picture of performance.

- The huge amounts of data generated make it difficult to derive the most decisive data. The issue is that there is so much data that the most telling information is obscured by the superfluous data - unable to see the wood for the trees.

- The tools used to generate the data have traditionally resided within IT departments and are paid for using IT budgets. Initially these tools were born out of the need for IT staff to ensure sufficient bandwidth and server capacity for their site(s). Often, the IT people are generally reticent about offering the data to those who arguably need it most marketing people.

- A corollary of the above problems is that the tools have been designed to be used and interpreted by the IT department, and without technical knowledge it has been extremely difficult for marketing people to construe any meaning from the outputs. Meaningful interpretation of the data is prevented because of the amount of extraneous data and the inability to visualise the data.

- There is a tendency to use data that are readily available (usually as standard reports) rather than trying to obtain data that answer key questions about performance. Each site has different propositions, goals and success measures; hence the reason why standard reports 


\section{Page hits are} misleading

\section{Revenue-based metrics}

are limited. These standard reports do not capture the complexity of human behaviour, and the questions posed by marketers are typically more intricate than standard reporting can respond to.

- People/departments are reluctant to put metrics in place to measure the success (or lack thereof) of an e-commerce venture. This is because these people or departments are responsible for the success of the site and are likely to be held accountable should it be determined that the venture is not quite as successful as it should be.

- A lack of clear definitions to measure site traffic. Measurements based on hits, impressions or page views are often ambiguous, and drawing conclusions about performance based on these data is difficult. The study by Forrester Research found that 98 per cent of site owners use site traffic, such as hits and unique visitors, to gauge performance. ${ }^{2}$

One can identify with a certain degree of accuracy what companies your visitors are from, what country they are from, how long visitors are spending on your site, which pages visitors are viewing (including the most frequently requested pages), the referring pages (the page from which a user linked through to your site) and which pages visitors exit the site from. While these are all useful data, they are insufficient for making key business decisions.

Data based on page hits are misleading. Each page on a website is made up of a number of components, including the text, the images and other types of files. Therefore when a user views one page on a site he/she may generate ten hits. Consider the following two scenarios.

- John visits amazon.com and accesses multiple pages of a site in a vain attempt to find a product. He leaves having failed to buy the product he was interested in.

- Tom visits bol.com, finds the book he is interested in very quickly and makes his purchase.

According to the site manager who subscribes to the theory that page hits are a good measure of site success, John is a more valuable customer than Tom.

Organisations are embarking on costly and time-consuming development (or redevelopment) projects without any consideration of the effect on the bottom line of the business. There is an urgent requirement for revenue-based metrics with which to gauge website performance. While the metrics above are useful (albeit limited), they are certainly not the most important. The key metrics are those that gauge whether or not business objectives are being met or not. For example, it is useful to know that you are attracting 10.2 million hits a month, but a far more telling metric is how many visitors actually make a purchase.

\section{Key metrics}

These revenue-oriented metrics can be numerous depending on the level of detail required. They can be categorised as macro- and micro-level metrics. Macro-level metrics describe the overall functioning of the site, 


\section{Beyond rudimentary measures}

whereas micro-level metrics are those that are specific to areas of the site or customer groups. Metrics are always quantifiable and expressed either as a value or a ratio. Table 1 summarises the macro metrics that an online retailer may use. These metrics and the figures associated with them provide data on the true state of the business and allow the retailer to identify changes in these figures. For example, the very low conversion rate for Product 1 hints at a problem area. One can hypothesise about the particular cause of the problem, such an inadequate product details, ambiguous product pricing or an unclear product image.

Tools and products, referred to collectively as Web analytics tools, are available on the market (Table 2) that allow organisations to measure against these metrics and build a detailed picture of website performance. They go beyond rudimentary measures, such as hits, to offer an insightful and thorough view of customer behaviour on the site. These tools make it easier to extract information - identifying patterns, potential problem 'hot spots' and correlations - and filter out superfluous information. The

Table 1: Example metrics for an online retailer

Number of unique visitors

Ratio of unique visitors to registered users

$2,280,000$

Ratio of unique visitors to buyers

Ratio of first-time vs repeat buyers

Frequency of visit

Duration of visit

Depth of visit (number of pages viewed)

Purchases per visit

Purchases per customer

Order value (basket size)

Abandonment rate ratio

Conversion rate by product group

Product Group 1

Product Group 2

Product Group 3

Product Group 4

Product Group 5

Conversion rate by product view

Product 1

Product 2

Product 3

Product 4

Product 5
$35 \%$

$12 \%$

$85 \%$

$1.6 \mathrm{pcm}$

$18.16 \mathrm{mins}$

68.4

4.2

6.72

$£ 44.38$

$32.81 \%$

$22.44 \%$

$46.80 \%$

$19.06 \%$

$92.88 \%$

$50.37 \%$

$10.47 \%$

$35.94 \%$

$93.14 \%$

$73.62 \%$

$61.37 \%$

Table 2: Web analytics vendors

\begin{tabular}{|l|l|}
\hline Accrue Software & NetIQ \\
Buystream & NetValue \\
Clickstream & RedEye \\
Coremetrics & SteelTorch Software \\
CustomerCentric & Visual Insights \\
Digimine & WebSideStory \\
Elytics & WebTrends \\
Intellitracker & Whitecross \\
Mtracking & \\
\hline
\end{tabular}


Aberdeen Group defines Web analytics as the 'monitoring and reporting on website usage so that enterprises can better understand the complex interactions between Web visitor actions and website offers, and leverage that insight to optimise the site for increased customer loyalty and sales'.

Understanding how a site is performing is only part of the issue, however, and the information derived is not necessarily actionable. A focus on usability facilitates intelligence-driven change to the site to impact directly on these key metrics.

\section{The significance of usability}

The Internet has gone beyond brochureware and there is now a proliferation of complex Web applications developed by organisations to meet the needs of their customers. This consequently increases the amount of interactivity between users and the sites. Usability is the extent to which this interactivity is accomplished effectively, efficiently and satisfactorily.

To understand usability is to consider the number of times you have been frustrated by your inability to find information on a site, got lost on a site because you do not understand its structure or been unable to buy a

28 per cent failed transactions

\section{Even Amazon has poor usability} product because you cannot navigate the checkout process. The Boston Consulting Group found in research that 28 per cent of all consumers attempted transactions failed, ${ }^{4}$ while a study by Zona Research found that 62 per cent of online shoppers had given up at least once while looking for products. ${ }^{5}$

Even the best-known sites are capable of committing usability crimes. In tests carried out on the Amazon site it was found that people would click on the 'back' button up to 30 times simply to return to the

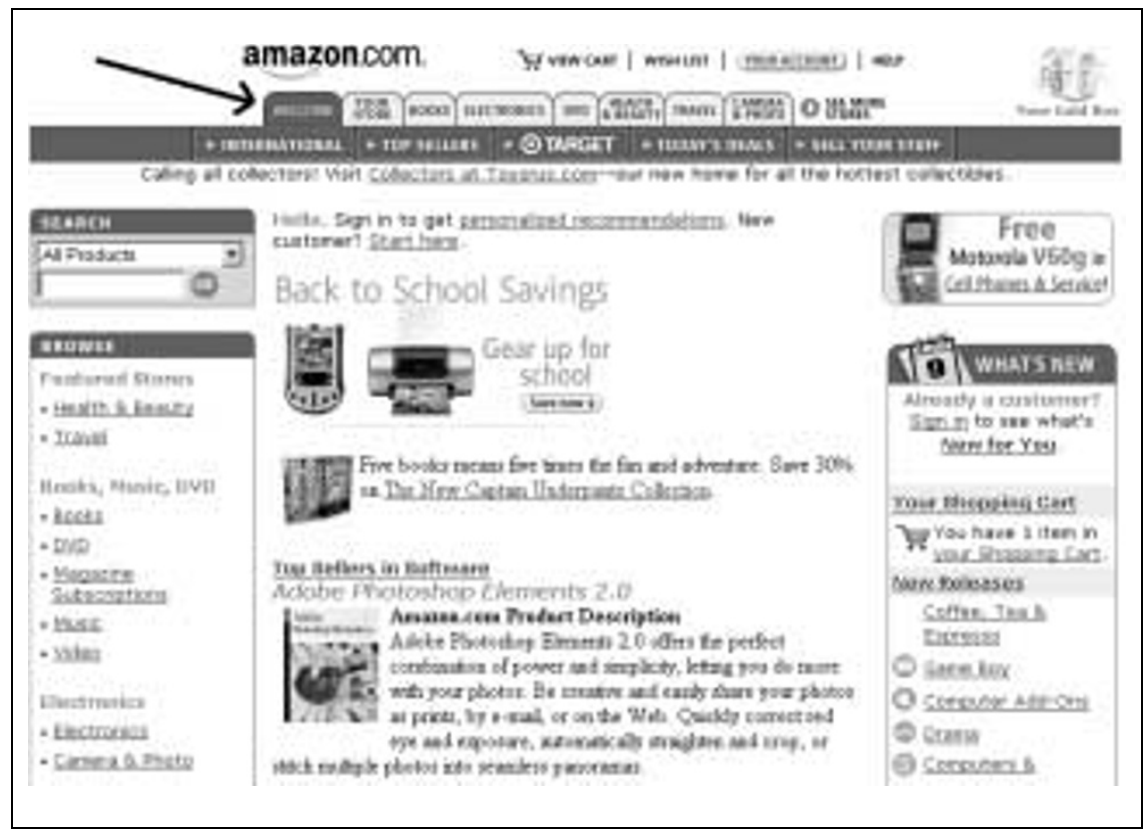

Figure 1: Amazon.com homepage 


\section{Reflect human behaviour}

homepage. The reason is simple: although the site provides a link back to the homepage (the 'welcome' link at the top left of Figure 1), this link is not labelled 'home'. People have become familiar with the convention of having a link labelled 'home' to return to the homepage, and this causes usability issues on the Amazon site.

\section{Misalignment of business and customer goals}

People come to sites to achieve goals. These goals, of course, vary according to the site and can include finding information, subscribing to receive information and buying products or services. In most circumstances the users' goals correspond directly to business goals. However, the issue is in how users want to achieve these goals and how organisations prescribe that users should achieve them. The reasons for this incongruence are manifold. In some cases the technical solution lacks flexibility to accommodate changes to the interface, while in other cases there are political reasons for this intransigence and of course budget considerations also constrain organisations. However, by far and away the primary reason is a lack of understanding of how users are using and would like to use the site.

The key is not in trying to alter human behaviour (a more than difficult undertaking), but in designing the site to reflect this behaviour. Ultimately it is far easier to change the design of a site than to modify the behaviour of the huge numbers of people using it. The focus should be on building a comprehensive understanding of why people are behaving in a certain way, and using this knowledge to provide a design more attuned to people's needs. This information may also offer the potential for predicting future behaviour.

A good case in point is banner advertisements. Many sites persist in using banner advertisements despite the fact that they have very little evidence to indicate whether they work or not. Marketers will tell you they work by pointing to the huge numbers of visitors clicking through on the advertisements. However, in isolation these numbers are meaningless because they cannot be compared to other scenarios and therefore make it difficult to perform relative judgments. The placement, size and appearance of these advertisements are determined more by an individual's idea of what they perceive to be a compelling advertisement rather than by hard facts about how site visitors perceive them. There is of course a great debate between those who believe that banner advertisements are viable and those who insist that most experienced Internet users exhibit 'banner blindness, 6 rendering banner advertisements ineffectual. The truth is that banner advertisements are appropriate for some sites but not for others. The point is that site owners rarely ascertain whether they are appropriate for their offering — they do not measure using eyetracking or other usability techniques.

\section{Real customer empowerment}

No other medium offers such control to the user as the Internet. Decisionmaking responsibilities now reside firmly on the side of the user, and these decisions include whether or not to go beyond the homepage, read 


\section{Users are extremely fickle}

the site's promotional copy, request product information, make a transaction and return to the site in the future. The time-sensitive nature of using the Internet and the proliferation in the number of competing offerings on the Internet mean that users are extremely fickle. A site that does not satisfy these irresolute users has very little hope of meeting its business objectives. While the medium may have changed the fundamental business principles still apply. Yet many organisations design sites that are at complete odds with the abilities, the needs, the motivations and the thought processes of people.

It will no longer suffice to pay lip service to customer service. The Web and this transfer of empowerment to the target audience have elevated the need for the provision of truly customer-centric Web applications. Customer-centric information is core to the planning and implementation of an e-business strategy. Companies are attempting to adopt a customercentric strategy but are impeded by their ability to determine what it is that people want. Now, possibly for the first time, organisations are compelled to acquire a veritable understanding of user needs and react to these needs efficiently.

\section{Usability methodologies}

Understanding user needs and behaviour is achieved through usability methodologies. 'Usability' is also shorthand for the approaches used to make a site easy to learn, easy to use and perform such that users will be satisfied with it. These specialised techniques are numerous, and include methods for organising site content and analysing the adherence of the site to accepted usability principles. The most frequently referred to method is usability testing, whereby a sample of site users are asked to complete a number of tasks and are observed by usability specialists attempting to pinpoint usability issues. These usability specialists are highly trained individuals, usually with a background in psychology.

Surveys or online questionnaires can also be used to understand if people are satisfied with their site experience. These tools can elicit important information regarding satisfaction levels and perceptions of the brand. However, this information should be treated with caution: while such self-reporting provides an insight into satisfaction levels it is inherently unreliable when understanding actual human behaviour. Witness the scenario in which a user fails to complete the simplest of tasks on a site yet subsequently gives the site a rating of nine out of ten in a survey. People find it extremely difficult to articulate actual experiences (whether good or bad) so, while useful, these data need to be tempered against observed behaviours in usability studies.

\section{The benefits of usability Increase revenue}

Customers who cannot buy will not buy. Convoluted e-business sites can lose many visitors and sales opportunities if customers cannot find the information or products they are interested in. Devising a positive user experience means that people are more likely to become customers 
Positive user experiences
Align with KPIs because they are able to do the things they want to do in a simple and straightforward fashion.

\section{Reduce customer churn}

A site that is high on usability is attractive to a customer as it allows him/ her to meet his/her objectives in a quick and simple manner. These sites are likely to encourage customers to return because they have had a positive user experience. A site lacking usability is likely to be visited just once - with all the competing offerings on the Internet a user is unlikely to expend much time and effort trying to understand a cumbersome site.

\section{Reduce support costs}

When a site fails to meet the information needs of a customer he/she will either opt to go to a competitor site or choose to use another, more expensive, channel. Servicing customers via an Internet site is accomplished at a fraction of the cost of other channels. Therefore, providing an experience that meets needs is likely to reduce the incurred cost of expensive channels.

\section{Strengthen brand equity}

In the absence of a sales person or traditional attributes of a brand the usability of the site effectively becomes the brand. Having had a positive user experience people are likely to hold the brand in higher regard.

\section{The problem with usability}

Usability may offer increased revenues, reduced support costs and competitive advantage. Positioning the customer at the centre of an e-commerce initiative by implementing a usability strategy is no longer enough, however. Organisations believe in the benefits of usability but find it increasingly difficult to justify increases in expenditure because they lack validation to warrant such an increase in investment.

With all of the best will in the world the intention is rarely to develop a site that makes users happy. Ultimately ingraining usability into the offering is about one (or both) of two things: increasing revenues or reducing costs. The difficulty is in measuring the benefits of usability and determining if these translate into real and tangible business benefits.

The tendency has been to consider usability as residing in the amorphous area inhabited by graphic design - more subjective than objective. This means that the impact of usability has been inherently difficult to measure (at least numerically), and in times of budget constraints there is a mounting pressure to demonstrate the ROI or, like other strategies, it is shelved.

Usability practices and strategies need to be more closely aligned with business objectives and key performance indicators (KPIs) or metrics. This means expressing the impact of usability in quantifiable terms. Correlating numbers with the benefits of usability increases the buy-in quotient within the organisation. 


\section{Understanding behaviour}

\section{Opportunity to improve}

\section{Visitors who buy}

\section{The new approach}

The problem with even the best of the Web analytic tools is that they simply tell you what is happening, but not why it is happening. For example, in the case of high shopping basket abandonment it is clear what the problem is but not why such abandonment is occurring. One e-commerce site justified the existence of a search facility on the site on the basis of the amount of traffic going to the search page. However, on closer examination it became apparent that the majority of users were leaving the site at the search results page, clearly indicating how ineffective the search feature was. Usability studies can offer a means of understanding why such behaviours are occurring.

The data collected and presented by the Web analytic tools allow organisations to make inferences about where usability issues are occurring. Where the success rates of user goals fall below 100 per cent (and they almost always do) there is an opportunity to improve the site. For example, if the data indicate that there is a very high abandonment rate at the shopping basket page then the usability efforts are focused on this area to understand the reasons why. Isolating problems areas in this manner allows an organisation to direct resources more reliably and cost effectively. Usability studies will ultimately reveal whether the high attrition rate is due to an unclear navigation scheme, terminology issues, privacy/security concerns or other factors.

With the ability to understand what is happening on your site, combined with the ability to understand why these things are happening, it is possible to develop a strategy that establishes how problems can be resolved.

To bring this to life, consider the role of marketing and marketing spend. Marketing dominates a commanding share of e-business budgets, and marketing/advertising spend is often the single largest investment organisations make in their sites. Marketing is charged with instilling a desire to visit the site, but what happens when this desire is poorly satisfied? Website traffic increases but sales do not.

Heavy marketing spend does not necessarily correlate with increased sales. The resources expended in generating traffic are often not cost effective and frequently exceed the revenue contribution. The poor sales performance is often a direct consequence of poor usability and even visitors with a propensity to buy do not (or cannot!), resulting in unfavourable conversion rates.

It is far more cost effective to increase the number of visitors who buy than to increase the number of visitors. There can be no arguing that generating visitor traffic to the site has to be a core concern, but converting these visitors into purchasers is fundamental to e-business success. Increasing the conversion rates also helps to justify increases in customer acquisition costs. It is difficult to explain high customer acquisition costs if the conversion rate is low. Returning to the example earlier, even if you vastly increase marketing activities the conversion rate will not rise above 12 per cent. Making improvements to the site, however, will increase this rate. Figure 2, an illustration provided by the Boston Consulting Group, ${ }^{7}$ shows how conversion rates are affected. 


\section{There are always metrics}

\section{Driven by business or marketing}

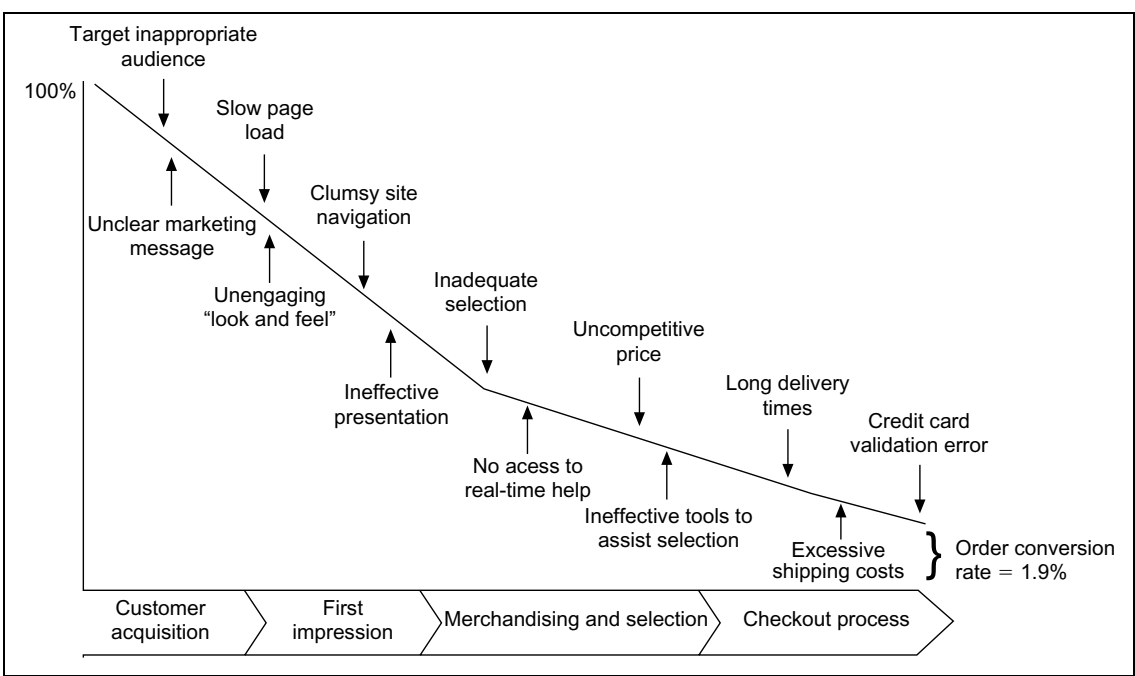

Figure 2: Conversion rate illustration

Note that metrics do not have relevance only for retail sites, but are applicable within almost every industry. The metrics may differ, but there are always metrics. For example, a financial services organisation may want to understand the abandonment rate of an online application form for a particular financial product, or a news provider may want to measure the page dwells on particular areas of the site. In the case of the financial services organisation a KPI could conceivably be the number of application forms received. By investigating the usability of the form, pinpointing pitfalls and rectifying these to ensure the forms are easier to complete, usability directly influences the KPI.

The optimal approach is to gauge metrics continuously and, when they do not look favourable, to investigate the problem by applying usability methods. Having isolated specific issues, rectify them and revisit the metrics. This process is essentially iterative, beginning with the core metrics and fine-tuning with other ancillary metrics. This process is described below (see also Figure 3).

\section{The process}

\section{Identify the metrics}

This involves understanding the business objectives and user goals, followed by a mapping of user goals against the site objectives. All industries and sites have different metrics, but all sites can be gauged using metrics. For example, for a site providing information (such as www.bbc.co.uk) a key metric would be page views. The development of these metrics should be driven more by business or marketing personnel rather than IT, and should ideally take the form of a workshop so that key stakeholders are involved.

\section{Configure tools}

Following the workshop the existing analytic tools should be configured in order to yield the data for these metrics. This may involve a number of 
Decrease abandonment rate

\section{Ongoing improvements}

tools offering parts of the data, or for some organisations procuring a new tool to meet the requirements.

\section{Benchmark the data}

Having configured the tools, take measurements of current performance for all of the various metrics. This will provide a benchmark of data to compare against future performance. Goals should be set at this stage to meet the business objectives. For example, for a retail site a goal may be to decrease the abandonment rate from 42 per cent to 20 per cent.

\section{Usability tests}

The benchmark data will point to problem areas that require analysis. For example, if the data suggest that the abandonment rate is particularly high during the checkout process then the checkout process should be investigated for usability issues. By observing a number of test participants using the site it will be possible to hone in on the problem areas (ie those areas that detract from the usability of the site).

\section{Implement changes}

Having found problem areas, design changes should be suggested to rectify the issues. Implementation of these changes should be done in a controlled way so that the effect of the changes can be isolated and measured. An example here might be the registration process. The site owners may make changes in accordance with the findings of the usability research and then monitor the number of visitors completing the registration process versus the baseline number before the changes.

\section{Collect data}

When the changes have been in place for a specific time period (on a busy site a week will suffice), measurements should again be taken of the metrics. These measurements should be compared against the baseline established from the benchmark data to determine if an improvement in the site's designated metrics has occurred. Again, registration serves as a good example. Suppose an e-commerce site had a registration conversion rate of 33 per cent (ie out of every 100 visitors to the site 33 are completing registration) and this increased to 42 per cent following changes - it is clear that the changes have had positive impact.

This process is iterative, with a constant emphasis on improving the metrics over time. This strategy allows for the creation of a dynamic feedback loop where learning about customer behaviours can be used to make ongoing improvements to the site.

This may now seem like a common-sense approach, but Jupiter found that 71 per cent of sites do not analyse site abandonment rates even though 66 per cent of consumers reported having abandoned a purchase during the process. ${ }^{8}$

\section{Conclusion}

This paper has detailed an approach to Internet strategy with the emphasis firmly on business objectives and the means to measure whether these 


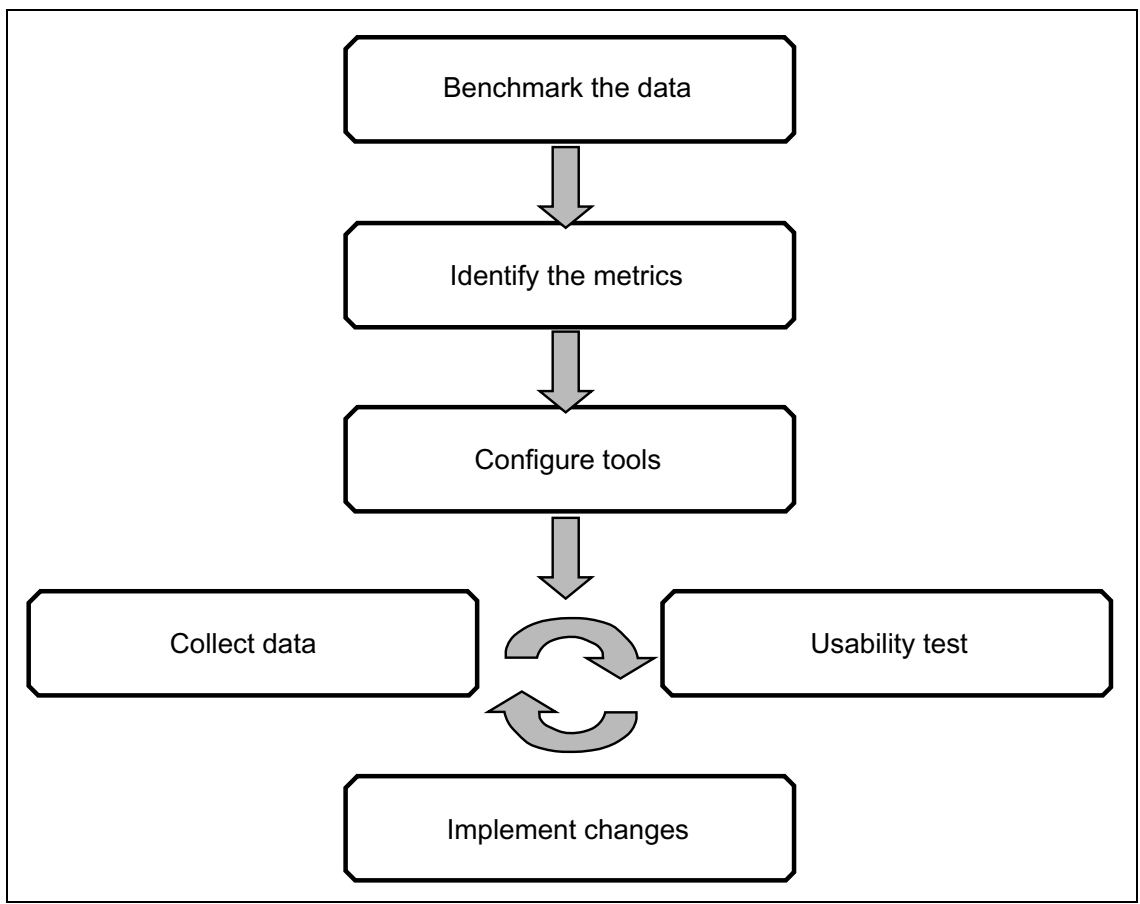

Figure 3: The process

\section{Impact the bottom line}

objectives are being met. Marketers will be able to rest assured that hardearned budgets are well merited while continuously enhancing their Internet offering.

Coupling the intelligence proffered by Web analytic tools with the ability to identify and remedy issues effectively by employing usability methods is a cost-effective strategy for Internet managers. While recognising the risk involved in introducing accountability for site success, the opportunity that an ROI-driven strategy represents should be attractive to those ultimately responsible for impacting on the bottom line of an organisation.

\section{References}

1. Forrester Research (2001) How To Measure What Matters, Forrester Research.

2. Ibid.

3. Aberdeen Group (2002) Web Analytics: Making Business Sense of Online Behavior, Aberdeen Group, Boston, MA

4. Boston Consulting Group (2000) The Canadian Online Retailing Report, BCG, Boston, MA, available at www.retailcouncil.org/research/cdnOnlineRetailing.pdf.

5. Zona Research (1999) Zona Research's Online Shopping Report, Zona Research, Redwood City, CA.

6. Benway, J. P. and Lane, D. M. (1998) 'Banner blindness: Web searchers often miss 'obvious' links', internetworking, December, http://internettg.org/newsletter/dec98/banner_blindness.html.

7. Boston Consulting Group, ref. 4 above.

8. Jupiter Research (2002) Web Site Analytics: Aligning Measurement and Business Goals to Drive ROI, Jupiter Media Metrix, Darien, CT. 